\title{
Anaerobic bacteria in routine urine culture
}

\author{
JOHN T. HEADINGTON AND BARBARA BEYERLEIN
}

\author{
From the Department of Pathology, the University of Michigan, and the Department \\ of Microbiology in the University of Michigan Medical Center, Ann Arbor, Michigan, U.S.A.
}

SYNOPSIS One hundred and fifty-eight anaerobic organisms from 147 patients were isolated from 15,250 consecutive clean mid-stream or catheter urine specimens. The pathogenicity of the anaerobic genera commonly isolated from urine is reviewed and discussed. Failure to establish anaerobic isolates as pathogens and a paucity of reported cases proving anaerobic bacteria as significant causes of urinary tract infection permitted discontinuation of anaerobic culture as part of the routine screening procedure for investigation of urinary tract infections.

Qualitative and quantitative urine cultures, as screening tests in the laboratory diagnosis of urinary tract infection, have become well-established procedures. To date, however, most studies have investigated the recovery of aerobic bacteria. This report summarizes the results of our attempt to isolate and identify anaerobic organisms. Anaerobic bacteriuria is assessed for clinical significance.

\section{MATERIALS AND METHODS}

SPECIMENS Routine cultures were done on 15,250 consecutive clean mid-stream or catheter urines received in the Clinical Microbiology Laboratory of the University Hospital from April 1962 to April 1965. Specimens were received from both in-patient and out-patient units; however, no attempt was made to separate specimens by source. Clinical records from 147 patients, representing 158 anaerobic isolates, were reviewed for evidence of urinary tract infection and the possible pathogenic role of anaerobic bacteria.

TEST METHODS In evaluating large numbers of routine cultures, test methods were simplified to provide generic rather than specific identification.

One-tenth millilitre of undiluted well-mixed urine was inoculated directly into $9.9 \mathrm{ml}$. of thioglycollate broth. Inoculated tubes were incubated at $37^{\circ} \mathrm{C}$. and examined daily for macroscopic growth for a maximum of eight days. All tubes revealing growth or questionable growth were Gram-stained and inspected for cellular morphology and Gram-reaction.

All Gram-negative bacilli were subcultured on MacConkey's agar for aerobic incubation at $37^{\circ} \mathrm{C}$., on $5 \%$ human blood agar for incubation in $3 \%$ carbon dioxide at $37^{\circ} \mathrm{C}$. The Gram-negative variably pleomorphic rods, which failed to grow on MacConkey's agar in 24 hours, did not grow on blood agar within 48 hours

Received for publication 20 June 1966. in $3 \% \mathrm{CO}_{2}$, but did grow anaerobically producing very small, round, slightly raised, translucent or transparent colonies, regarded as Bacteroides species. This group of organisms was not further identified.

Non-sporulating Gram-positive rods of characteristic morphology which produced poor to slight growth in thioglycollate broth and no growth within $\mathbf{4 8}$ hours on $5 \%$ human blood agar, incubated in $3 \%$ carbon dioxide or under anaerobic conditions, were classified as Lactobacillus species.

All Gram-positive cocci were subcultured to $5 \%$ human blood agar plates and incubated aerobically, capneically, and anaerobically for $\mathbf{4 8}$ hours. The Grampositive cocci which grew only under anaerobic conditions were identified either as Peptostreptococcus species or Peptococcus species on the basis of morphological characteristics after Gram-staining of thioglycollate cultures and on the presence or absence of catalase production. Species characterization was not done.

Catalase-negative Gram-positive rods growing anaerobically were considered to be Clostridia. The majority of these organisms were further characterized specifically on the basis of routine tests.

One hundred and ninety-five of 15,250 consecutive anaerobic urine cultures yielded one or more anaerobic isolates. Of these 62 , or $32 \%$, of anaerobic isolates were present in pure culture.

Although mixed anaerobic and aerobic cultures predominated, there were only rare instances of more than one anaerobic organism isolated per specimen. These results are summarized in Table $I$.

Table II indicates the frequent occurrence of anaerobic genera in routine urine culture. Lactobacillus species were in the majority followed by the Clostridial group. Bacteroides, Peptostreptococcus, and Peptococcus species together composed less than $16 \%$ of the total.

The ratio of isolation of anaerobes in females as compared to males, $3 \cdot 7 / 1 \cdot 0$, is at variance with the usual University Hospital in-patient out-patient ratio of about $1 \cdot 0 / 1 \cdot 0$. Although this indicates the greater likelihood of 
TABLE I

ISOLATION OF ANAEROBIC BACTERIA FROM ROUTINE CULTURE

\begin{tabular}{lrc} 
& $\begin{array}{l}\text { Total } \\
\text { Nos. }\end{array}$ & $\begin{array}{l}\text { Percentage } \\
\text { Total }\end{array}$ \\
\hline Cultures studied & 15,250 & \\
Anaerobic isolates & 195 & $1 \cdot 3$ \\
Anaerobes isolated in pure culture & 62 & $0 \cdot 4$ \\
Anaerobes isolated in mixed culture & 133 & $0 \cdot 9$
\end{tabular}

TABLE II

FREQUENCY OF ISOLATION OF ANAEROBIC GENERA

\begin{tabular}{lrrrr} 
Species & Pure & Mixed & Total & $\begin{array}{l}\text { Percentage } \\
\text { Total }\end{array}$ \\
\hline Lactobacillus & 39 & 67 & 106 & $54 \cdot 6$ \\
Clostridium & 5 & 52 & 57 & $29 \cdot 3$ \\
Bacteroides & 5 & 9 & 14 & $7 \cdot 3$ \\
Peptostreptococcus & 7 & 4 & 11 & $5 \cdot 7$ \\
Peptococcus & 6 & 0 & 6 & $3 \cdot 1$
\end{tabular}

recovering an anaerobe from females, the nearly ubiquitous presence of anaerobic lactobacilli in the female genital tract after the menarche and before the menopause appears to be primarily responsible for this difference.

\section{DISCUSSION}

LACTOBACILLUS Lactobacillus species have rarely been proved primary pathogens. However, anaerobic stains causing pulmonary abscess (Korttila, 1953) (single case) and subacute bacterial endocarditis (Marschall, 1938) (single case) have been described. No example of urinary tract infection due to anaerobic Lactobacillus species has been reported.

One hundred and six strains of anaerobic Lactobacilli were isolated in this study, 39 in pure culture and 67 in mixed culture. No attempt was made to differentiate human vaginal strains.

The clinical records for patients with pure lactobacilluria were reviewed, and in no instance could an anaerobic Lactobacillus species be incriminated as a urinary tract pathogen. Twenty-two of 53 isolates from mixed cultures were associated with clinical findings suggestive of urinary tract infection. The remainder were recovered from asymptomatic patients.

The common association of lactobacilluria and diabetes mellitus was an interesting finding. The details are illustrated in Figure 1. In brief, lactobacilluria in a male of any age except in the newborn period, or in a woman 50 years old or older was associated with diabetes mellitus in approximately $50 \%$ of cases. Presumably, increased tissue glucose is the altered ecological factor of greatest significance.

CLOSTRIDIUM In spite of numerous published

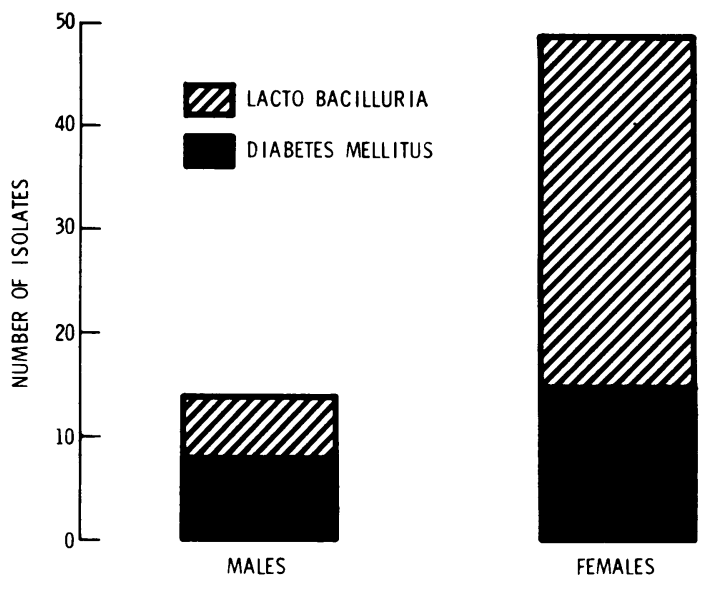

FIG. 1. Lactobacilluria and diabetes mellitus.

reports of clostridial infections of soft tissues of the pelvis and retroperitoneum, no example of primary infection of the urethra, urinary bladder, ureter, or kidney was found. On occasion, however, the initial locus of infection becomes a moot point because wounds in these structures offer a suitable portal of entry to contiguous tissue. Nevertheless, in the absence of surgical or traumatic wounds, Clostridial infections of the urinary tract must be considered rare.

Clostridial species were the second most common 2 anaerobes isolated. Members of this genus accounted $\overrightarrow{\vec{B}}$ for $29.3 \%$ of all anaerobic isolates. Fifty-one of $57 \mathrm{~g}$ isolates were classified as Clostridium perfringens; one as Clostridium septicum; and one as Clostridium sporogenes. Four could not be further identified.

In this study there were no specific infections of the urinary tract which could be directly attributed to these organisms. A single non-fatal case of postoperative clostridial septicaemia occurred following transurethral resection of the prostate gland without evidence of pelvic infection.

BACTEROIDES Bacteroides species, said to outnumber Escherichia coli as normal flora of the gastro- $N$ intestinal tract are, however, infrequent pathogens. Following ingress through portals of entry in the $N$ nasopharynx, gastrointestinal, or female genital tract Bacteroides infections usually produce suppurative foci in soft tissues or bones and may or 0 may not be associated with septicaemia. In clinically $\frac{C}{D}$ significant infections the concomitant isolation of $\stackrel{\oplus}{+}$ another organism, usually a micro-aerophilic or 7 anaerobic streptococcus, is found in about onequarter to one-half of cases.

Bacteroides species rarely have been proved $\vec{\otimes}$ urinary tract pathogens: a single case of pyelo- 
nephritis with calculus formation in which a Bacteroides species was isolated from urine in pure culture (Beigelman and Rantz, 1949); a report of Bacteroides funduliformis isolated in pure culture from a perinephric abscess (Smith and Ropes, 1945); a single case report of mixed Bacteroides fragilis and anaerobic streptococcal infection from a patient with unilateral pyelonephritis (Schulte, 1939).

McVay and Sprunt (1952) reported seven isolations of Bacteroides species from urine, including four in pure culture, but were unable to demonstrate these as significant urinary tract pathogens. The experience of these authors closely parallels our own. A review of the clinical records from nine patients from whose urine a Bacteroides species was isolated failed to establish the pathogenicity of these organisms.

Seven of the nine isolates were from females. This may reflect the greater chance for contamination of the distal urethra in the female by genital or faecal flora compared with the male.

PEPTOSTREPTOCOCCUS The pathogenicity of the anaerobic streptococci is well established, particularly in post-partum infections or as occasional pulmonary pathogens. In concert with Gramnegative anaerobes or staphylococci they can produce severe chronic suppurative inflammation in soft tissue. Anaerobic streptococci, isolated in pure culture, as a primary cause of pyelonephritis or cystitis is unreported. A single case report (McVay and Sprunt, 1952) describes repeated recovery of an anaerobic streptococcus and Bacteroides species from the urine with recovery of the same bacteria from tissue.

Anaerobic streptococci were recovered from the urine on 11 occasions, four in mixed culture and seven in pure culture. A review of clinical records failed to relate these organisms to urinary tract disease.

PEPTOcoccus Anaerobic Gram-positive cocci classifiable as anaerobic staphylococci were isolated in pure culture on six different occasions from six different patients. Five of the six isolates were from females. This finding would be in agreement with Bergey (Breed, Murray, and Smith, 1957) that the usual source of these organisms is the female genital tract if they be regarded as contaminant flora of the urethra.

The significance of anaerobic staphylococci as urinary tract pathogens is uncertain at best. No recorded examples of these organisms acting as urinary tract pathogens were found and they could not be considered pathogenic in any patient in this small group.

CLINICAL INFECTION AND ANAEROBIC BACTERIURIA The clinical records of patients who had clear-cut signs and symptoms of urinary tract infection and also had anaerobic bacteriuria, were selected for additional study. Fifty-four patients could be so classified. Of these, only seven yièlded anaerobic species in pure culture. Of the remaining 47 an acceptable aerobic pathogen predominated or no conclusion could be drawn. In those cases from which a possible aerobic pathogen was also isolated, the distribution and incidence of aerobic genera was the same as for those urine specimens not containing an anaerobic organism, i.e., Escherichia coli, coliform bacteria, enterococci, Aerobacter-Klebsiella species, proteus species, and pseudomonas species. In considering the possibility of anaerobic infection in the seven symptomatic patients with anaerobic bacteriuria yielding an organism in pure culture the following criteria for pathogenicity were set:

(1) Isolation of the same anaerobic organism directly from the inflammatory process; (2) presence of an infection characteristic for the organism isolated; (3) two or more consecutive urine cultures yielding the same anaerobe in pure culture or as the predominating organism. In no instance could these criteria be satisfied either singly or in combination. The clinical details are summarized in Table III. These findings indicate that although anaerobes may occasionally be isolated in pure culture from patients

TABLE III

URINARY TRACT SYMPTOMS AND ANAEROBIC BACTERIURIA

\begin{tabular}{lllll} 
Patient & Age & Sex & Organism & Urinary Tract Disease \\
\hline 1 & 40 & Male & Cl. perfringens & Congenital polycystic kidneys \\
2 & 19 & Male & Cl. perfringens & Chronic glomerulonephritis \\
3 & 70 & Male & Lactobacillus & None \\
4 & 52 & Female & Peptostreptococcus & Vesicle neck contracture \\
5 & 73 & Female & Bacteroides & Transitional cell carcinoma, bladder \\
6 & 36 & Female & Lactobacillus & Chronic pyelonephritis \\
7 & 57 & Female & Peptostreptococcus & None
\end{tabular}

${ }^{1}$ Patient had diabetes mellitus. 
with proved urinary tract abnormalities, solid corroborative evidence for significant anaerobic infection is difficult to obtain.

ANAEROBIC CULTURE AS A SCREENING TEST The failure to establish the pathogenicity of a single anaerobic isolate after review of 147 patients with urine cultures positive for anaerobic species and the paucity of acceptable reports proving these organisms as significant urinary tract pathogens has permitted the conclusion that an attempt to isolate and identify anaerobes in urine culture as a routine is not justifiable as part of a screening procedure. The discontinuation of routine anaerobic culture was therefore effected without loss of confidence in conventional quantitative culture for detection of significant bacteriuria.

In an effort to define more clearly the possible role of anaerobic bacteria in urinary tract infection, anaerobic culture of urine is done randomly for investigation of specific diagnostic problems and as routine on premium specimens obtained by percutaneous bladder puncture or ureteral catheterization.
It should be emphasized that the screening methods applied to the cultures reported in this study $\overrightarrow{\vec{\rho}}$ are not sufficiently exacting for maximum recoveryof anaerobic and capnophilic bacteria. Small등 numbers of fastidious organisms are, on occasion, $\overline{\bar{c}}$ undoubtedly overgrown by less demanding organ- $\bar{\nabla}$ isms in fluid media. Primary cultures should be done on highly nutrient solid media in Brewer anaerobic ${ }^{\infty}$ jars or anaerobic incubators in an atmosphere of $\vec{O}$ line gas or nitrogen plus $10 \%$ carbon dioxide for 48 hours at $37^{\circ} \mathrm{C}$. It is also important to use fresh specimens to maintain optimal conditions and to Gram-stain films of sediment as a cross-check for. faulty incubational technique.

\section{REFERENCES}

Beigelman, P. M., and Rantz, L. A. (1949). Arch. intern. Med., 84,음 605 .

Breed, R. S., Murray, E. G. D., and Smith, N. R. (1957). In Bergey's Manual of Determinative Bacteriology, 7th ed., edited by $Z$ R. S. Breed et al., p. 423. Williams and Wilkins, Baltimore. Korttila, K. (1953). Ann. Med. exp. Fenn., 31, 22.

Marschall, F. (1938). Zbl. Bakt., 1 Abt. Orig., 141, 153.

McVay, L. V. Jr., and Sprunt, D. H. (1952). Ann. intern. Med., 36 56.

Schulte, T. L. (1939). Proc. Mayo Clin., 14, 536.

Smith, W. E., and Ropes, M. W. (1945). New Engl. J. Med., 232, 31 